\title{
Kleine Mittheilung für die Geschichte der Operation des grauen Staars.
}

\author{
Von \\ Dr. Joh. Bapt. Ullersperger, \\ vormal. herzogl. Seuchtenbers'schem Leibarzte.
}

\begin{abstract}
Der Anfang der Geschichte der Staaroperation ist in Dunkel und Mythe eingehüllt. Die ältesten Traditionen vermögen nicht ersteres zu erhellen und letztere beginnt erst mit den alten Griechen.

Bekanntlich haben die Israeliten während ihres Aufenthaltes in Aegypten Wissenschaften und Künste von den Aegyptern erlernt und manches, ja man darf wohl sagen vieles davon in die weite Welt getragen; namentlich waren es die Leviten, welche Vieles sich aneigneten. In späterer Folge wissen wir, dass selbst die Griechen den Aegyptern grosse Geschicklichkeit im Heilen und auch in der Augenheilkunde zugestanden haben. Diesen Ruf scheinen sich die „Juden“ als Ophthalmiatriker noch zu den Zeiten der Ptolemäer und der berühmten Alexandrinischen Zeitperiode erhalten zu haben. Man behauptet indessen, dass die Alexandrinische Schule die "Depression des grauen Staars" aus Asien hatte und dieselbe wesentlich verbesserte.
\end{abstract}


Die Mythe der Operation des grauen Staars beginnt mit den Griechen, namentlich mit der Ziegen-Mythe. Es wollten nämlich die Alten beobachtet haben, dass sich die Ziege von der bei ihr nicht so selten vorkommenden i zióyvo dadurch befreite, dass sie sich einen Dornstachel ins Auge sticht, wodurch die getrübte Augenflüssigkeit ausfliesst. Plinius erzählt, dass sie sich durch Einstechen einer Stachelbinse davon befreie und Antiphilus beschreibt dieses in einem Epigramme. In jedem Falle wird man versucht, die in die Periode der Araber fallende SuctionsOperation des grauen Staars darauf zu beziehen. Auch scheinen hiemit die Synonyme der Krankheit selbst in einigem historischen Zusammenhange zu stehen wie cata-

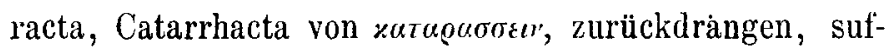

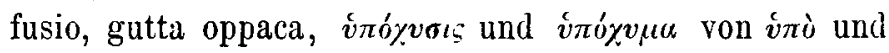
$\not \dot{v} \varepsilon v$, suffundere, weil man die Entstehung der Krankheit einer Trübung der Augenflüssigkeiten zuschrieb.

Die darauf gegründete Annahme, dass die SuctionsMethode älter sei als die Depression, scheint uns nicht wahrscheinlich, wenn man sich anders an die literarischen Docunente hält. Als geschichtliche Hypothese hat die Sache freilich die Ziegenmythe für sich und dic nosogenetische Analogie, indem, nach Entfernung der trüben Flüssigkeit durch Aussaugen, Sehvermögen hergestellt werden konnte. Der dritte historische Grund, die perforirten Staarnadeln zur Zeit der Araber, wodurch diese gleichfalls die dunklen Körper durch Aussaugen entfernten, scheint uns eher die gänzliche Rückkehr zur Depression zu beweisen, nachdem man die Extraction verlassen hatte, dennoch aber die Entfernung des das Sehen hindernden Elementes nicht plötzlich aufgeben wollte, vielleicht schon darum, weil man doch von den Resorptions-Thätigkeiten der Theile noch schwache Begriffe hatte.

Einige Schriftsteller nehmen an, dass zu Zeiten des 
Hippokrates von der Operation les grauen Staars noch nichts bekannt gewesen sei, während Hecker*) annimmt, dnss zu dieser Zeit die Niederdrückung des grauen Staars von umherziehenden ungebildeten Chirurgen geübt und ausgebildet worden ist.

Soviel scheint historisch alle:dings richtig, dass zur Zeit der Trennung der Medicin von der Chirurgie in Alexandrien sogar Laien auftauchten, welche sich ausschliesslich mit Ophthalmiatrik befassten und auch die dahin einschlägigen Operationen verrichteten.

Da sehr geprüfte and fachkundige Arbeiter, wie Stephanus Hieronymus de Vigiliis von Creutzenfeld in seiner bibliotheca chirurgica, der unter oculorum morbi**) alle Schriftsteller aufführt von den alten Griechen an bis Ende der ersten Hälfte des achtzehnten Jahrhunderts; ferner der berühmte Haller in seiner bibliotheca chirurgica schon vor ihm ***), in zweiten Jahrzehnt des laufenden Jahrhunderts Friedrich Jäger aus Kirchberg in seiner Dissertation, $\dagger$ ) endlich auch noch A. G. van Onsenoort in seiner Geschichte der Augenheilkunde $\dagger$ ) und J. W. L. Gründer †††) in seiner Geschichte der Chirurgie

*) Er schreibt wenigstens in Rust's Handbuch der Chirurgie Berlin und Wien 1831 8. IV. Bd. p. 620: „Wahrscheinlich ist schon zu dieser Zeit, wenn nicht früher, die Niederdrückung des grauen Staars, deren Ursprung nicht angegeben werden kann, üblich gewesen; doch ist anzunehmen, dass sie nur von ungebildeten umherziehenden Chirurgen ausgeübt worden.

**) Bibliotheca chirurgica etc. Stephani Hieronymi de Vigiliis von Creutzenfeld. Vindobon. 1781. 4. Tom. II p. 1246.

***) Bibliotheca chirurgica Basil. 1774. 4. Tom. I.

†) Fried. Jäger Kirchbergens., de Keratonyxidis usu. Viennae 1812, die auch Just. Radius in vol. I seiner scriptor. ophthalmolog. minor. Lips. 1826. 8. p. 150 wiedergegeben hat.

$+\dagger)$ Geschichte der Augenheilkunde als Einleitung in das Studium derselben vou A, G, van Onsenoort. Aus dem Holländischen von Wutzer. Bonn 1838. 8 .

††) Geschichte der Chirurgie. Berlin 1859. 8. 
theils historische Lücken gelassen über die Geschichte der Staaroperation, theils verschiedene Angaben gemacht haben, - so glaubten wir diesem Zweigchen der Heilkunst schuldig zu sein, jeden kleinen Beitrag dazu der Vergessenheit entreissen zu müssen. Die meisten Autoren haben aus den unvergänglichen Werken Hallers geschöpft, und um unsre kleine unbedeutende literarische Beigabe an ihr Plätzchen einreihen zu können, wird es nöthig, obenerwähnten Autoren in Kürze so viel zu entlehnen, dass damit einiger Zusammenhang erreicht wird. Unter den Griechen citirt Haller Lathyrion "de cataractae depositione". Eigentlich bezeichneten die Griechen diesen operativen Akt mit $\mu \varepsilon \tau u q \dot{q} \sigma \omega$, depositionem per acum, die

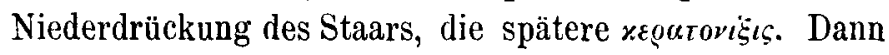
führt er Symon oder Symeon an, qui docet quando cataracta apta sit deponi.

Die wichtigste Rolle in der alten Geschichte der Operation spielt indess unstreitig die arabische Periode, welche ich besser maurospanische nennen möchte, weil die árztlichen Celebritäten geborene Spanier waren.*) Unter ihnen kamen auch Juden vor und darum können wir dem Ausspruche van Onsenoort's nicht beipflichten, wenn er schreibt: „Es ist zu verwundern, dass bei einer Nation (sollte wohl heissen Volk) wie die Juden nirgendwo ausdrückliche Erwähnung der Augenheilkunde geschieht, es ist wenigstens denkbar, dass sie mit den Augenübeln und einigen darauf bezüglichen chirurgischen Operationen nicht unbekannt waren." Wir werden den Beweis in einer historischen Thatsache nachbringen. Bei Avicenna lesen wir: „nonnullos disrumpere inferiorem partem corneae et extrahere aquam per eam tunicam." Er missbilligt die Extraction durchaus als gefährlich und giebt der Depression den Vorzug. Abulcasis operirte

*) Averrhoes, Avicenna, Rasis sind in Cordova geboren. 
durch Depression unica punctione vel duabus et duobus instrumentis. Nach der Operation gab er ein hypnoticum. Er sah auch den Staar auf medicamentösem Wege heilen, auch beobachtete er Wiederaufsteigen desselben. Rhazes*) erwähnt, dass man sich in seiner Gegend Irak Bagdad 1499 einer myrtenblattförmigen Nadel bediene, durch deren Höhlung man den Staar aussauge. Es bestanden demnach um jene Zeiten die Operation durch Depression, was die allgemeinste Methode war, der Hornhautstich oder Hornhautschnitt mit Suction oder mit völliger Extraction. Dem Dogmatiker Antyllus, von dem wir lesen: "Primus post Christum uatum non solum propositae, verum reapse factae cataractae extractionis meminit, hancque cataractam operandi methodum, quamdiu ista parva est, commendat, majorem autem, quin et oculi humores simul profluerent, extrahi non posse, autumans ..." standen als Opposition zwei be= deutende arabische Autoritäten entgegen, Avicenna und Avenzoar, welche beide die Extraction als gefährlich verwarfen. Da man nun von Albucasis auch liest, dass er und seine Anhanger , acum perforatam excogitaverunt, per cujus foramen, postquam jam ingressa esset, suctu cataractam extraxerunt," so characterisirt sich mit diesen historischen Belegen demnach die Zeitperiode der maurospanischen Chirurgen als Eigenthümerin der Operation per suctionem et per depressionem, welche letztere über erstere weitaus das Uebergewicht behielt. Wir heben diese geschichtliche Thatsache darum hervor, weil sich unsere gleich nachfolgende Mittheilung genau derselben anreiht.

Guy von Chauliac, gewöhnlich Guido de Cauliaco genannt, citirt von Cana Musali de Baldach, dass er auf beiden Augen den Staar deprimirt habe und dass er sich

*) Edit. Vinet. 1509 libr. II tr. VI cap. 2 pag. 50. 
viele Mühe gegeben mit Herausgabe der chaldäischen und hebräischen Bücher der Aerzte.*) Unbestritten hat demnach die Operation im Orient und im maurischen Spanier eine erste historische Rolle gespielt; denn im Occident schrieb Johann de Gaddesden (John of Gastiden oder Gatisden) in seiner rosa anglica: „adparet neminem eo aevo medicorum et chirurgorum cataractam scivisse deponere, quare ermahnt wird, man solle sich an Hunden dazu einüben. Und Balescon de Tharane vulgo Valescus de Taranta notirt in seinem philonio, wo er von den Augenkrankheiten handelt: "medicos honoris sui studiosos cataractae depositionem non adgredi, et junioribus circumforaneis relinquere.

Vico oder de Figo, von Einigen Joanctinus Vigus genannt, beschreibt in seiner practic. CLII die Depression des grauen Staars, angebend, dass er sie von umherziehendeu Staarstechern habe verrichten sehen.

Ambroise Paré (Ambrosius Paraeus) deprimirte den grauen Staar mit einer stählernen Nadel (acu ad cataractam planiuscula scindente).

Es scheint nun, dass in Deutschland die Operation des grauen Staars so ziemlich umherziehenden Oculisten und Ophthalıniatrikern überlassen war, die wie Stein-und Bruchschneider herumzogen, **) bis auf Georg Bartisch, geboren $1535 \mathrm{zu}$ Königsbrück***) und chursächsischer Hof-Oculist. Auch er hatte die Staar-Operation handwerksmássig gemacht und sich aus der niedern Chirurgie emporgehoben. Das Erscheinen seines Werkes, das wir

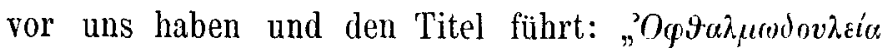
oder Augendienst oder Bericht von Ursachen aller Schäden,

*) Zu finden in der Collectio chirurgica Vincta 1497 und 1499. 2.

**) Man vergleiche nur, was wir eben von Vigo in Italien und von Paré in Frankreich angeführt.

***) S. das nähere Bibliographische bei Isensee 1096-97, bei Gründer Geschichte der Chirurgic. Bıeslau 18j9. 8. p. 337. 
Gebrechen, Mängeln der Augen und des Gesichtes, wie man solchen anfänglich mit gebürlichen Mitteln begegnen, vorkommen und wehren, auch wie man solche Gebrechen künstlich durch Arzney, Instrument und Handgriff curiren, wirken und vertreiben sol. mit schönen herrlichen, contrafectischen Figuren, der Anatomia Beyde des Haubtes und der Augen etc. durch Georg Bartisch von Königsbrück, Bürger, Oculist, Schnit und Wundartzt in der Churfürstlichen alten Stadt Dressilen im 1583 Jare in 2. In dem vierten Teil wird angezeigt und beschrieben von den Innerichen Cataracten der Augen, so in gemein der Star und Hirnfelle genannt werden. 42. 6.

In dem fünfften Theil wird angezeigt und beschrieben, wie man den rechten, zeitigen und reiffen Star künstlich durch die Handgriffe und Instrument wirken und stechen, Auch solche Patienten mit der Cur und Heilung recht und wohl versehen und versorgen sol. Und hat dis Teil in sich zehen Capitel. 56. 6."

Häser bemerkt darüber ganz richtig: "Seine Schrift bezeichnet ebenso deutlich den Zustand, in den er die Augenheilkunde antraf, als die Fortschritte, welche dieselbe ihm selbst verdankt." Diesem Ausspruche fügen wir nur hinzu, dass dieses zunachst nur auf Deutschland zu beziehen sei.*)

Nun theilt uns Don Juan de Ferreras in seiner historia de España. syclo XV. 10 parte. Madrid 1722 in $4^{0}$ p. 218 mit: „Dass Abiabar, Rabiner aus Lerida, berühmter Arzt, Chirurg und Astrolog an König Don Juan von Aragonien im Jahre 1468 am 12. September die Staar-Operation verrichtet habe."

*) Die Literatur bat Aug. Andrii in seinem Grundriss der gesammten Heilkunde 1 Thl. Leipzig 1846 sehr ausführlich und mit seltenem Fleiss und grosser Fachkenntniss gesammelt. Es möchte wohl das vollständigste sein, was über Cataracten-Literatur besteht p. $99-118$ $\S 65$ v. J. 1532 an bis 1845 . 
Seine Worte sind: quien le passò la aguja en el ojo derecho, y le quitò de el las cataractas, y riendo el buen efecto de la opcracion, de allì à un mes, contra la sentencia de el medico, hizo que le ejecutasse el remedio en el ojo izquierdo, que tuvo el mismo feliz efecto, con que quidò el Rey libre de aquella molestia. (Er führte ihm die Nadel ins rechte Auge ein und nahm so den Staar hinweg. Nachdem el sich nach Ablauf eines Monats von dem guten Erfolge der Operation überzeugt hatte, führte er, selbst gegen die Meinung des Leibarztes, dieselbe Operation auch am linken Auge aus, welche gleich glïcklichen Erfolg hatte und wodurch der König von scinen Augenleiden gänzlich befreit wurde.)

Wir fassen das Interesse, welches diese Aufzeichnung für die Geschichte der Staar-Operation hat, da dieselbe noch in kein literarisches Organ aufgenommen ist, in folgende Punkte zusammen: 1) dass die Staar-Operation unter den Arabern und Mauro-Spaniern, namentlich die Depression, ihren historischen Glanzpunkt feiert vom Alterthume her bis zum Mittelalter. 2) Dass diese Zeit und dieser Ort die Ausgangspunkte geworden sind für die Ausbreitung nach den Lander-Radien des übrigen Europa's. 3) Dass die Operation von Juden verrichtet worden so gut wie von Arabern und Mozarabes (MauroSpaniern). 4) Endlich, dass die Staar-Operation in jenen Zeiten nicht, wie die meisten Historiographen geschrieben, blos von umherreisenden Staarstechern geübt worden, sondern auch von erprobten und berühmten Chirurgen. 